\title{
Espacialização da leishmaniose tegumentar na cidade do Rio de Janeiro
}

\author{
Spatial distribution of tegumentary leishmaniasis \\ in the city of Rio de Janeiro
}

Helia Kawa 1,2

Paul o Chagastell es Sabroza 2

\footnotetext{
1 Secretaria Estadual de Saúde do Rio de Janeiro. Rua México 128, Rio de Janeiro, RJ 20031-142, Brasil. hkawa@uol.com.br 2 Departamento de Endemias Samuel Pessoa, Escola Nacional de Saúde Pública, Fundação Oswaldo Cruz. Rua Leopoldo Bulhões 1480, Rio de Janeiro, RJ 21041-210, Brasil. sabroza@ensp.fiocruz.br
}

\begin{abstract}
The authors analyze the historical and spatial determinants of the implantation, persistence, and spread of cutaneous leishmaniasis in the municipality of Rio de Janeiro and the links between the disease and the organization of urban space and occupation processes on the periphery of the city beginning in the early 20th century through the 1980s. A pattern of outbreaks that was restricted and spatially discontinuous but sequenced was observed and seen to be linked to a dynamic process of urban real estate appreciation, comprising a large endemic area for cutaneous leishmaniasis. An analysis of the occupation and organization of urban space in the so-called Western Zone of Rio de Janei ro was conducted, considering new functions of spatial el ements expressed through changing work relations, land use, and land value. Urbanization of the area produced the necessary conditions to intensify an endemic pattern of well-defined outbreaks where human mobility and the work process increased contact between susceptible individuals and vectors. Analysis showed spatial units with differentiated risk levels. Key words Leishmaniasis; Disease Outbreaks; Spatial Analysi s; Zoonosis; Epidemi ology
\end{abstract}

Resumo Os autores analisam os determinantes históricos e espaciais da implantação, persi stência e difusão da lei shmani ose tegumentar na ci dade do Rio de Janei ro, e sua arti culação com os processos de organização e ocupação do espaço urbano na periferia da cidade, do início do século até o final da década de oitenta. A análise da distribuição dos surtos epi dêmicos neste período, mostrou a presença de um conjunto de focos descontínuos, delimitados no tempo e no espaço e articulados pela dinâmica de valorização da terra urbana, constituindo uma grande zona endêmica de lei shmaniose tegumentar caracterizada por unidades espaciais com riscos diferenciados da endemia. O modelo utilizado foi a análise do processo de ocu pação e organização do espaço na periferia da cidade, consi derando as novas funções dos el ementos espaciais expressos através de diferentes relações de trabalho, uso do solo e valor da terra. O movimento de urbanização criou as condições necessárias à intensificação da endemia em focos bem definidos, onde o processo de trabal ho possi bilitou maior contato entre indi víduos suscetíveis e vetores. Palavras-chave Leishmaniose; Surto de Doenças; Análise Espacial; Zoonoses; Epidemiologia 


\section{Introdução}

A leishmaniose tegumentar (LT) é uma endemia que vem mostrando, nas décadas recentes, um aumento expressivo no número de casos, assim como uma importante difusão espacial (MS, 2000).

Uma das observações que mais chamou a atenção neste período foi o registro da transmissão em áreas de ocupação antiga e, inclusive, em espaços urbanos, contrariando a expectativa de que, com o aumento da pressão antrópica e a conseqüente eliminação dos focos naturais, esta endemia deveria ter sua importância reduzida (Pessoa \& Barreto, 1948).

Revendo a literatura, Valim (1993) descreve três padrões de transmissão da LT no Brasil. Um padrão silvestre, localizado na região Amazônica e no Centro-Oeste brasileiro, no qual a transmissão ocorre em focos naturais em ecossistemas florestais. Um segundo padrão, também em focos naturais com ciclo silvestre, mantido em áreas florestais preservadas como rugosidades em áreas de produção agrícola tradicional, como ocorre no Nordeste nos estados do Ceará e da Bahia e, na Região Sudeste, em Minas Gerais e no Rio de Janeiro. E, ainda, um terceiro padrão de transmissão, cuja ocorrência tem sido observada nas periferias de áreas urbanas.

Deve ser destacado que sob a denominação de ciclo urbano foram agregadas áreas com características distintas, que parecem configurar situações particulares na transmissão da endemia: aquelas onde ocorreu a expansão da cidade nos limites da floresta, como foi observado em $\mathrm{M}$ anaus onde o vetor é silvestre e o risco decorre da distância entre o indi víduo e a mata (Andrade, 1998); áreas com preservação de pequenos trechos com cobertura florestal preservada no interior do espaço urbano, com possibilidade de exposição eventual do indivíduo ao parasita neste ambiente restrito (Gomes \& Neves, 1998) e, finalmente, áreas em que o processo de urbanização criou as condições favoráveis à produção de focos da endemia, como na cidade do Rio de Janeiro, assegurando a persistência e reprodução ampliada da leishmaniose tegumentar (Kawa, 1998).

Nesta cidade, casos autóctones de LT vêm sendo registrados de forma descontínua, desde a primeira década do século XX. O primeiro registro foi efetuado por d'Utra-e-Silva em 1915.

Em 1922, ocorreu uma epidemia na localidade de Águas Férreas, no bairro do Cosme VeIho, na região central da cidade, estudado por Cerqueira \& Vasconcellos (1922), que foi a primeira descrita em área urbana no Brasil.
Após um período silencioso de cerca de cinqüenta anos, a LT reapareceu no Rio de Janeiro, já agora na Zona Oeste da cidade, e, a partir daí, passou a apresentar uma importante difusão espacial, inclusive para municípios vizinhos como Niterói, Mangaratiba, Nova I guaçu (Oliveira Neto et al., 1988; Soares et al., 1995). Estudos epidemiológi cos real izados nos focos detransmissão da Região M etropolitana do Rio de Janeiro, puderam caracterizar um padrão, conjunto de características epidemiológicas e fatores de risco, bastante diferente daquele observado nos trabalhos realizados nas frentes agrícolas. Dentre eles destacam-se: incidência igual por idade e sexo, ausência de risco ocupacional, baixa proporção de formas mucosas e acentuada agregação familiar dos casos (Sabroza, 1981). O parasita isolado nos casos humanos foi sempre a Leishmania braziliensis (Grimaldi Jr. et al., 1989).

Os levantamentos entomológicos mostraram que o flebotomíneo encontrado com mais freqüência nesses focos foi a Lutzomya intermedia, representando cerca de $90 \%$ dos animais capturados nas coletas realizadas nas proximidades do domicílio, seguida de Lu. migonei. A Lu. intermedia foi a única espécie encontrada infectada com L. braziliensis (Rangel et al., 1990) e é extremamente adaptada às áreas de transição situadas no limite entre o peridomicílio e as matas de segunda formação, como ficou demonstrado nos focos de São Paulo e do Rio de Janeiro (Gomes, 1979; Gomes \& Neves, 1998; Lima, 1986; Rangel et al., 1990).

A ocorrência das doenças endêmicas depende tanto das características biológicas dos elementos que participam no ciclo de transmissão como de determinantes históricos, sociais e ambientais (Albuquerque, 1993; Sabroza et al., 1992).

Diversos pesquisadores destacam a relevância do espaço socialmente organizado como uma categoria essencial na investigação do processo saúde-enfermidade, lançando mão do conceito de espaço geográfico, utilizado pela Geografia Crítica, na análise da interação da sociedade com a natureza (Silva, 2000).

Na cidade do Rio de Janeiro, trabal hos importantes vêm procurando relacionar o movimento de espacialização dos processos de produção e reprodução social com as condições concretas de vida de grupos sociais particulares (Abreu, 1992; Corrêa, 1992; Ribeiro, 1997) e os modelos desenvolvidos têm trazido contribuições relevantes ao estudo dos padrões de endemicidade das doenças nas cidades brasileiras (Sabroza et al., 1995).

Este artigo tem como objetivo analisar os determinantes históricos e espaciais da implan- 
tação, persistência e difusão da LT no Município do Rio de Janeiro, e sua articulação com os processos de organização e ocupação do espaço urbano na periferia da cidade, do início do século até o final da década de oitenta.

\section{Material e métodos}

A série histórica de registro da LT no Estado do Rio de Janeiro foi iniciada em 1974, a partir do trabalho desenvolvido pelo Grupo de Estudo e Controle da Leishmaniose em Jacarepaguá, do Ministério da Saúde, cujos dados encontramse consolidados no Departamento de Endemias Samuel Pessoa, Escola Nacional de Saúde Pública, Fundação Oswaldo Cruz. Com o término das atividades desse Grupo, a extinta SUCAM (Superintendência de Campanhas de Saúde Pública, atual Fundação Nacional de Saúde), tornou-se responsável pelo registro, diagnóstico e tratamento da leishmaniose no Posto de Saúde de Vargem Grande, da Secretaria Municipal de Saúde do Rio de Janeiro, onde foram coletados todos os dados referentes à doença utilizados no presente estudo.

A cidade do Rio de Janeiro situa-se na Região Sudeste Brasil, no litoral atlântico sul, e ocupa uma área de $1.255,3 \mathrm{~km} 2$, com as seguintes coordenadas: latitude extremo norte 22o 45' $05^{\prime \prime}$, latitude extremo sul $23004^{\prime} 10^{\prime \prime}$, longitude extremo leste 430 06' 30" Wgr., longitude extremo oeste 430 47'40" Wgr. (IPLANRIO, 1995).

As informações populacionais foram extraídas do Anuário Estatístico da Cidade do Rio de Janeiro 93/94, editado pela Empresa Municipal de Informática (IPLANRIO, 1995). Os mapas foram adaptados a partir de figuras do mesmo Anuário.

A recuperação histórica referente à LT e à cidade do Rio de Janeiro foi feita através de revisão bibliográfica em instituições especializadas.

A metodologia adotada na análise dos dados e do processo de espacialização da leishmaniose tegumentar utilizou categorias da organização social do espaço urbano e da teoria de focos de doenças naturais (Pavlovsky, 1964), e modificados pela atividade humana (Rosicky, 1967; Sinnecker, 1976).

O conceito de espaço foi tomado de Santos (1988:26): “o espaço deve ser considerado como um conjunto indissociável deque participam, de um lado, certo arranjo de objetos geográficos, obj etos naturais e objetos sociais, e de outro, ... a sociedadeem movimento".

Para análise da ocupação do solo do Rio de Janeiro, utilizou-se o conceito de região urbana da escola da Geografia Crítica: “...região é uma enti dade concreta, resul tado de múl tiplas determinações, ou seja, da efetivação dos mecanismos de regi onalização sobre um quadro territorial já previamente ocupado, caracterizado por uma natureza já transformada, heranças culturais e materiai s e determinada estrutura social eseus conflitos" (Corrêa, 1987:46).

\section{Resultados}

O primeiro caso autóctone de LT no Estado do Rio de Janeiro foi registrado por Rabello em 1913.

Dois anos depois, d'Utra-e-Silva (1915) referiu a ocorrência da doença em al guns municípios e localidades do estado: Campos, Cantagalo, Itaocora, Itacuruçá, Macaé, Maricá, Mangaratiba, Porto das Flores, São José da Boa Morte e na cidade do Rio de Janeiro, nos bairros de Realengo, Jacarepaguá e Gávea.

Em 1922, ocorreu uma epidemia de cerca de cinqüenta casos em localidades próximas dos bairros de Santa Teresa e Cosme Velho, distantes apenas $4 \mathrm{~km}$ do centro da cidade.

Registros da época referem a ocorrência de casos esporádicos desde 1919 nesta mesma área (Cerqueira \& Vasconcellos, 1922).

Nesses períodos foram acometidos indistintamente homens, mulheres e crianças, principalmente aqueles que residiam perto da floresta, que havia sido preservada dentro do perímetro urbano. As pessoas atingi das não eram trabalhadores agrícolas, como havia sido observado nas epidemias registradas em outros estados (Aragão, 1922; Cerqueira \& Vasconcellos, 1922) em comparação ao observado na frentes pioneiras de São Paulo.

A grande maioria desses pacientes apresentava ulcerações comuns com aspecto característico das produzidas pela L. braziliensis. As lesões eram únicas ou múltiplas, localizadas nos membros inferiores, pescoço, face e cabeça, sendo raras no tronco. A proporção de doentes encontrados com lesão mucosa (três casos ou $11,6 \%$ ) foi considerada bastante reduzida em relação ao total, embora muito alta quando comparada com as freqüências atuais.

Na epidemia de 1922, Aragão (1922) demonstrou que flebotomíneos capturados no interior dos domicílios estavam infectados com leishmanias, e a espécie de vetor identificada foi o Phlebotomus intermedius, posteriormente considerada Lu. intermedia. Foram atingidas as localidades de Águas Férreas, onde estava o maior foco, além de Santa Alexandrina e Lagoinha, focos menores da doença, todos situados nas encostas da vertente atlântica do Maciço da Tijuca. 
Essa região apresentava características ambientais bastante particulares. Desde o ciclo de cultivo do café, no início do século XIX, sua vegetação primitiva havia sido muito devastada. Em 1856, devido à necessidade de proteger os mananciais que abasteciam de água a cidade, começou o processo de reflorestamento do Maciço da Tijuca (Abreu, 1992), onde se constituiria, anos depois, o Parque Nacional da Tijuca, a maior floresta tropical localizada numa área urbana.

Depois do surto em Águas Férreas em 1922, no limite entre a floresta e as áreas urbanizadas, não foram mais registrados casos de LT nos bairros próximos às várzeas e encostas do Maciço daTijuca.

Cinqüenta e dois anos mais tarde, surgiu uma nova epidemia no município do Rio de Janeiro (Sabroza, 1981), agora em Jacarepaguá, Zona Oeste da cidade, com 162 casos novos registrados, atingindo localidades situadas nas várzeas e encostas do maciço da Pedra Branca, próximo à estrada dos Bandei rantes, que corta a Região Administrativa (RA) de Jacarepaguá e a da Barra da Tijuca (Figura 1).

Na Tabela 1, encontra-se a distribuição dos casos de LT notificados no ano de 1974, por local de residência. Vargem Grande e Camorim, localizadas atualmente na RA da Barra da Tijuca, foram aquelas que apresentaram maior número de doentes.

Assim como na epidemia de 1922, a freqüência da LT foi a mesma em homens e muIheres, e $52 \%$ dos casos ocorreram em menores de 14 anos.

A incidência global da doença nessa área no ano de 1974, foi de 10 por mil habitantes, observando-se um risco muito maior nas localidades situadas nas várzeas e encostas (19,9 por mil habitantes) do que na baixada (1,4 por mil habitantes).

O estudo entomológico nos diversos focos, mostrou que a Lu. intermedia foi a espécie mais freqüentemente encontrada nos domicílios, peri-domicílios e abrigos de animais domésti$\cos (92,65 \%)$, seguida da Lu. migonei $(6,8 \%)$ (Sabroza, 1981).

O conjunto de investigações realizadas naquela oportunidade ressal tou a importância do domicílio como fator de risco na transmissão da LT em Jacarepaguá em 1974. Mostrou também, que as características epi demiológicas foram muito semel hantes àquelas da epidemia de 1922, embora a doença apresentasse uma distribuição bem mais ampla do que a observada na década de 1920.

Nos anos seguintes à epidemia de 1974 em Jacarepaguá, novos surtos epidêmicos de LT atingiram outras locali dades e bairros da cidade, também situados nas encostas e várzeas do Maciço da Pedra Branca, estendendo a área de endemicidade às encostas e vertentes das baixadas de Campo Grande e Guaratiba (Figura 2).

De 1975 a 1988, foram registrados mais 1.006 doentes de LT no Município do Rio de Janeiro, com uma média anual de 72 casos, que somados aos 162 pacientes diagnosticados em 1974, atingem um total de 1.168 notificações.

As características epidemiológicas dos pacientes nesse período foram novamente muito semelhantes àquelas encontradas nas epidemias de 1922 e 1974: a freqüência foi a mesma em homens e mulheres, e casos também ocorreram em menores de 10 anos de idade $(21,7 \%)$.

No entanto, verificou-se que ao contrário das epidemias anteriores, que se mantiveram circunscritas a focos definidos, no período de 1975 a 1988, a LT passou a apresentar um padrão de distribuição espaço-temporal caracterizado por sua difusão e propagação através de focos temporários. Na Tabela 2, observa-se a freqüência dos registros de LT em distintos períodos estudados, de 1975 a 1988, por local de residência, verificando-se que, em intervalos de tempo distintos, os doentes se concentraram em diferentes localidades.

A partir de 1975, as maiores proporções de casos foram observadas em Vargem Grande (15,5\%), bairro da RA da Barra da Tijuca (XXIV) e nas localidades Ilha de Guaratiba, Barra de Guaratiba e Estrada das Taxas, na RA de Guaratiba (Tabela 2).

Entre 1978 e 1980, Grumari e Piabas, situadas na RA da Barra da Tijuca (XXIV RA) e Pau da Fome, na XVI RA (Jacarepaguá), foram responsáveis por $18 \%$ e $24,3 \%$ respectivamente, dos casos (Tabela 2).

As localidades da RA de Campo Grande (Campo Grande, Rio da Prata, Cabuçu, Caboclos, Lameirão Pequeno e Viegas), contribuíram de maneira expressiva entre 1981 e 1984, somando juntas, aproximadamente, $27 \%$ dos doentes notificados. Nesse mesmo período, Vargem Grande e Camorim, localidades da RA da Barra da Tijuca, voltaram a apresentar as maiores proporções de doentes, registrando, cada uma, aproximadamente $13 \%$ de casos.

Nos quatro anos posteriores (1985 a 1988), Vargem Grande novamente registrou o maior porcentual de casos (20,5\%), seguida das localidades situadas na RA de Jacarepaguá (Pau da Fome, Rio Grande e Rio Pequeno), que foram responsáveis por $20,8 \%$ dos doentes notificados nesse período.

$\mathrm{Na}$ Figura 3, encontram-se as localidades que apresentaram mais de dez casos de LT no município do Rio de Janeiro, entre 1975 e 1988. 
Figura 1

Área atingida em 1922 e 1974 e principais focos* de leishmaniose tegumentar americana.

Município do Rio de Janeiro**, 1974

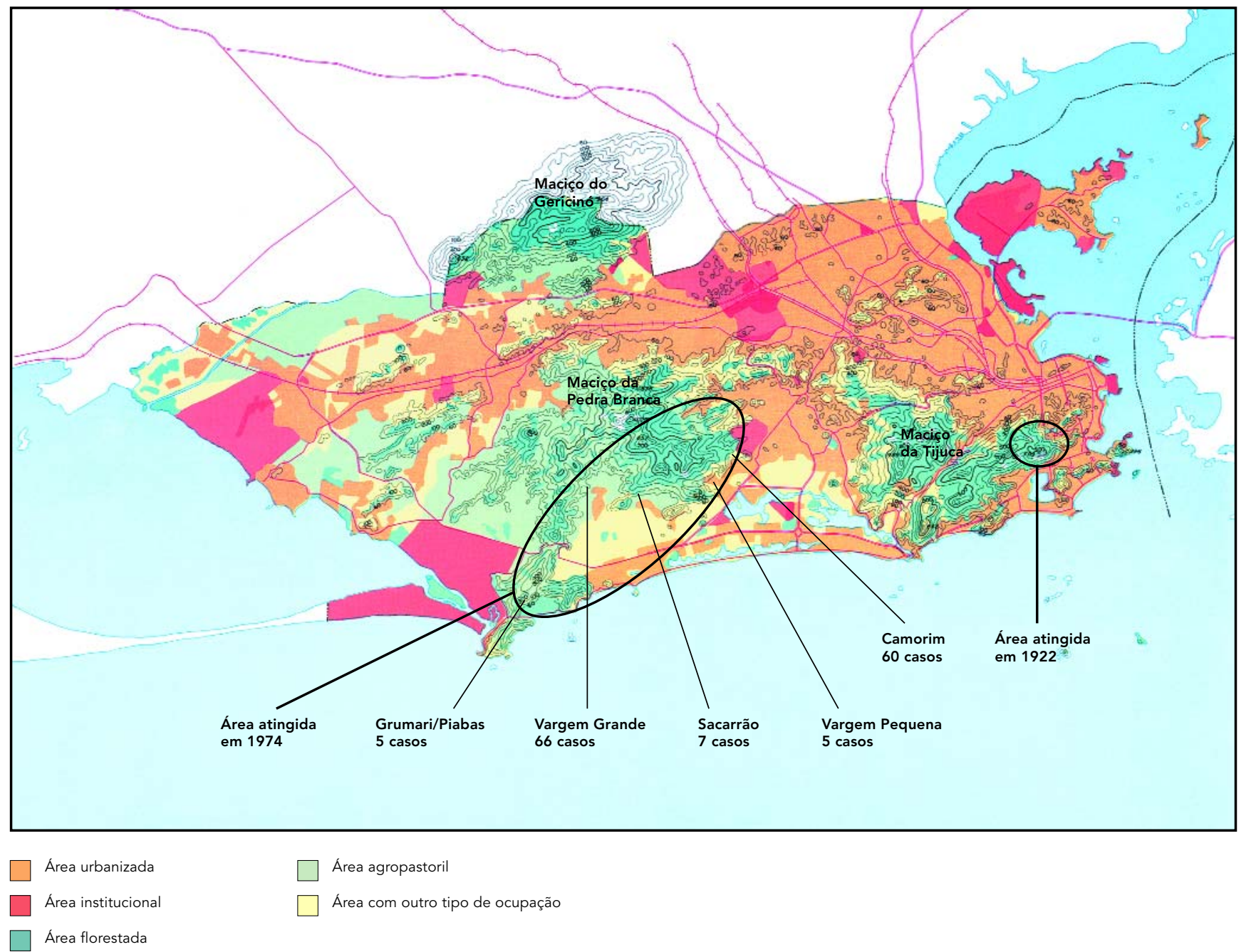

* Localidades que registraram cinco casos ou mais no período. Localização aproximada.

** Mapa adaptado do Anuário Estatístico da Cidade do Rio de Janeiro, 1993/1994. Mapa 17.

Comparando esta figura com aquela onde se encontram os focos de LT identificados em 1974 (Figura 1), pode-se observar que todas as localidades onde a enfermidade se manteve, estão situadas nas vertentes e várzeas próximas às encostas do Maciço da Pedra Branca.

Nota-se ainda, nessas mesmas figuras, a dispersão dos casos de LT pela Zona Oeste da cidade no período de 1975 a 1988, configurando a grande extensão da área endêmica do município. Observa-se assim, uma distribuição desigual da endemia caracterizada pela pre- sença de diversos focos descontínuos, intercalados por localidades onde ocorrem apenas casos isolados.

O comportamento do padrão de distribuição espacial indica que a dinâmica da localidade não pode ser analisada apenas a partir do lugar onde ela se encontra. Torna-se necessário situá-la no contexto da região onde está inserida, a partir de uma perspectiva integradora, ou seja, considerando sua dinâmica regional e a produção social do espaço onde a transmissão se materializa. 
A Tabela 3, apresenta algumas características das áreas endêmi cas de LT no Município do Rio de Janeiro, registrando diferenças relevantes. Onde as taxas de crescimento populacional eram elevadas como Riocentro e Recreio, os valores da densidade populacional líquida foram os mais baixos. Já em Campo Grande e, principalmente, em Bangu, verificaram-se altas densidades populacionais e baixas taxas de incremento populacional. Essa heterogeneida-

Tabela 1

Localidades da cidade do Rio de Janeiro que registraram cinco ou mais casos de leishmaniose tegumentar em 1974.

\begin{tabular}{lrr}
\hline Localidade & Casos & \multicolumn{1}{c}{$\%$} \\
\hline Camorim & 60 & 37,3 \\
Vargem Pequena & 5 & 3,1 \\
Vargem Grande & 66 & 41,0 \\
Sacarrão & 7 & 4,3 \\
Grumari/Piabas & 5 & 3,1 \\
Outros & 18 & 11,2 \\
Total & 161 & 100,0 \\
\hline
\end{tabular}

de no perfil demográfico das áreas endêmicas expressa momentos distintos no processo de ocupação urbana: enquanto Riocentro e Recreio apresentaram um processo de expansão, Bangu e Campo Grande já eram áreas consolidadas do ponto de vista da organização do espaço da cidade.

A relação entre essas duas variáveis (crescimento e densidade populacional líquida) com as taxas de incidência média de LT nas respectivas áreas endêmicas encontram-se nas Figuras 4 e 5.

Observa-se que as taxas de incidência média da LT no período estudado, foram muito mais elevadas em áreas endêmicas que se encontravam em processo de consolidação, como Riocentro e Recreio, que registraram respectivamente 45,46 e 18,26 por 10 mil habitantes. Em Bangu e Campo Grande, áreas endêmicas já consolidadas do ponto de vista urbano, a transmissão da endemia foi muito mais baixa $(0,65$ e 0,05 por 10 mil habitantes, respectivamente) apontando que a dinâmica do processo endêmico-epidêmico da LT no Município do Rio de Janeiro, guarda estreita associação com os processos particulares de ocupação da cidade.

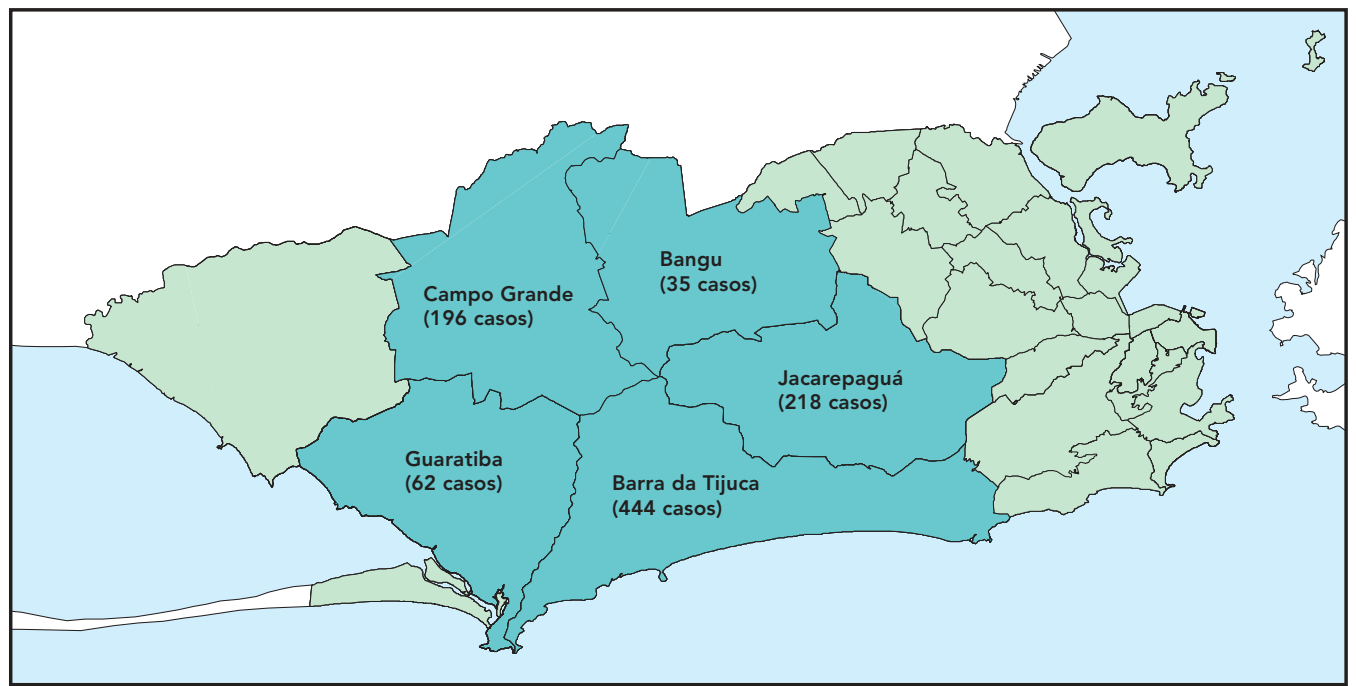

* Mapa adaptado do Anuário Estatístico da Cidade do Rio de Janeiro, 1993/1994. 
Distribuição dos casos de leishmaniose tegumentar por período de estudo e local de residência*. Município do Rio de Janeiro, 1975 a 1988.

\begin{tabular}{|c|c|c|c|c|c|}
\hline \multirow{2}{*}{$\begin{array}{l}\text { Região Administrativa/ } \\
\text { Local de residência }\end{array}$} & \multicolumn{5}{|c|}{ Período de estudo } \\
\hline & 1975-1977 & 1978-1980 & 1981-1984 & 1985-1988 & Total \\
\hline \multicolumn{6}{|l|}{ Barra da Tijuca } \\
\hline Camorim & 1 & 9 & 48 & 7 & 65 \\
\hline Vargem Pequena & 8 & 2 & 3 & 4 & 17 \\
\hline Vargem Grande & 16 & 19 & 49 & 65 & 149 \\
\hline Sacarrão & 3 & 1 & 3 & 3 & 10 \\
\hline Rio Bonito & 0 & 5 & 4 & 0 & 9 \\
\hline Grumari/Piabas & 6 & 31 & 11 & 3 & 51 \\
\hline \multicolumn{6}{|l|}{ Jacarepaguá } \\
\hline Pau da Fome & 8 & 42 & 40 & 33 & 123 \\
\hline Estrada Teixeiras & 6 & 2 & 30 & 6 & 44 \\
\hline Rio Grande/Rio Pequeno & 2 & 1 & 14 & 33 & 50 \\
\hline \multicolumn{6}{|l|}{ Campo Grande } \\
\hline Campo Grande & 6 & 1 & 24 & 22 & 53 \\
\hline Rio da Prata & 2 & 15 & 36 & 28 & 81 \\
\hline Cabuçu/Caboclos & 0 & 3 & 23 & 1 & 27 \\
\hline Lam. Pequeno/Viegas & 5 & 8 & 19 & 1 & 33 \\
\hline \multicolumn{6}{|l|}{ Bangu } \\
\hline Santíssimo/Senador Camará & 1 & 4 & 2 & 2 & 9 \\
\hline Serra do Bangu & 0 & 0 & 1 & 1 & 2 \\
\hline Realengo & 0 & 0 & 16 & 1 & 17 \\
\hline Teixeira Realengo & 0 & 0 & 0 & 7 & 7 \\
\hline \multicolumn{6}{|l|}{ Guaratiba } \\
\hline Barra de Guaratiba & 4 & 3 & 6 & 2 & 15 \\
\hline Ilha de Guaratiba & 21 & 8 & 4 & 4 & 37 \\
\hline Estrada Taxas & 8 & 0 & 0 & 0 & 8 \\
\hline Outros (fora da área endêmica) & 6 & 19 & 43 & 94 & 162 \\
\hline Total & 103 & 173 & 376 & 317 & 969 \\
\hline
\end{tabular}

* Santíssimo e Senador Camará são bairros vizinhos, situados em regiões administrativas distintas. Foram agregados num único local de residência, devido à disposição das áreas onde ocorreram os casos de leishmaniose tegumentar.

\section{Discussão}

Os trabal hos realizados nos focos de LT no Município do Rio de Janeiro e sua região de influência, nas últimas décadas, fundamentados nos modelos bio-ecológicos desenvolvidos pela linha de investigação da medicina tropical, mostraram reiteradamente a importância do lugar de transmissão e também identificaram os diferentes elementos que constituem o ciclo básico de produção e reprodução da LT, com exceção apenas dos seus reservatórios silvestres, ainda não identificados (Aguiar et al., 1996; Azevedo, 1984; Barbosa-Santos et al., 1998; Li- ma, 1986; Marzochi, 1985; Marzochi \& Marzochi, 1994; Pirmez, 1986; Sabroza, 1981; Soares et al., 2000; Toledo, 1987).

No entanto, todo esse conhecimento acumulado não permitiu explicar a produção, a persistência e a distribuição espaço-temporal dessa endemia na periferia da área urbanizada da cidade do Rio de Janeiro.

Aquelas variáveis, tão relevantes na descrição do lugar de transmissão da doença, mostraram-se insuficientes para compreender o conjunto de focos estruturados que constituíram a região de endemicidade de LT no Rio de Janeiro, trazendo a necessi dade da construção 


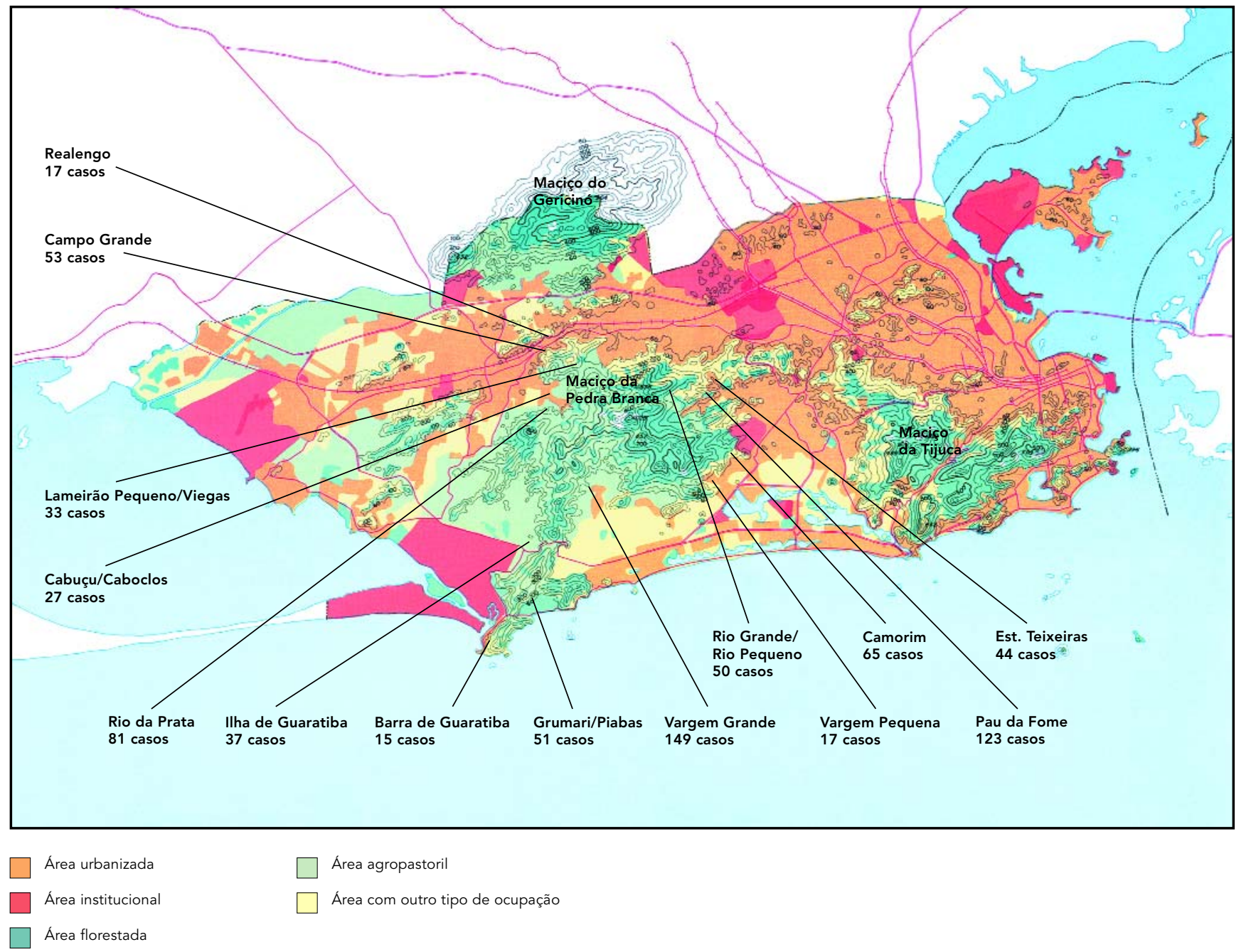

* Localidades que registraram dez casos ou mais no período. Localização aproximada.

** Mapa adaptado do Anuário Estatístico da Cidade do Rio de Janeiro, 1993/1994. Mapa 17

de uma perspectiva que considerasse o problema a partir de um nível de maior complexidade, permitindo recuperar a historicidade e a espacialização dessa endemia.

Quando se incorpora na análise do processo endêmico-epidêmico variáveis que permitam entender o espaço geográfico em transformação, conforme entendido pela Geografia Crítica, verifica-se que as condições necessárias à transmissão e difusão da LT foram criadas no processo de construção e organização do espaço urbano da cidade do Rio de Janeiro, particularmente na história da incorporação de sua Zona Oeste, iniciado desde meados do século XIX.

A transformação da terra agrícola em urbana se deu, em grande parte, devido ao processo de estagnação dos estabelecimentos rurais, tornando seu retal hamento em lotes, mais atraente do que cultivá-las. Como relata Ribeiro (1997: 227): “....as plantações das zonas periféricas da ci dade muito provavel mente enfrentam al tos custos, só tornando-se viáveis na base da baixa capitalização. Tais fatos tornarão interessantes a venda de chácaras, sítios e pequenas fazendas para fins de loteamentos, o que efeti- 


\section{Tabela 3}

Taxas de incidência média de leishmaniose tegumentar e características demográficas em áreas endêmicas do Município do Rio de Janeiro.

\begin{tabular}{lccc}
\hline Área endêmica & $\begin{array}{l}\text { Crescimento } \\
\text { populacional anual } \\
(1980 / 1991)\end{array}$ & $\begin{array}{l}\text { Densidade populacional } \\
\text { líquida, 1980 } \\
\text { (habitante/hectare) }\end{array}$ & $\begin{array}{c}\text { Taxa de incidência média* } \\
\text { Riocentro }\end{array}$ S,08 $^{\text {Recreio }}$ \\
Taquara & 6,85 & 0,8 & 45,46 \\
Campo Grande & 3,97 & 1,7 & 18,26 \\
Bangu & 2,86 & 17,2 & 1,24 \\
Guaratiba & 1,01 & 19,4 & 0,65 \\
\hline
\end{tabular}

* Taxas por 10 mil habitantes (1974 a 1988).

Fonte: Anuário Estatístico da Cidade do Rio de Janeiro 93/94 (IPLANRIO); Banco de dados do Departamento de Endemias Samuel Pessoa, Escola Nacional de Saúde Pública, Fundação Oswaldo Cruz.

\section{Figura 4}

Crescimento populacional e incidência média de leishmaniose tegumentar em áreas endêmicas. Município do Rio de Janeiro, 1974 a 1988.

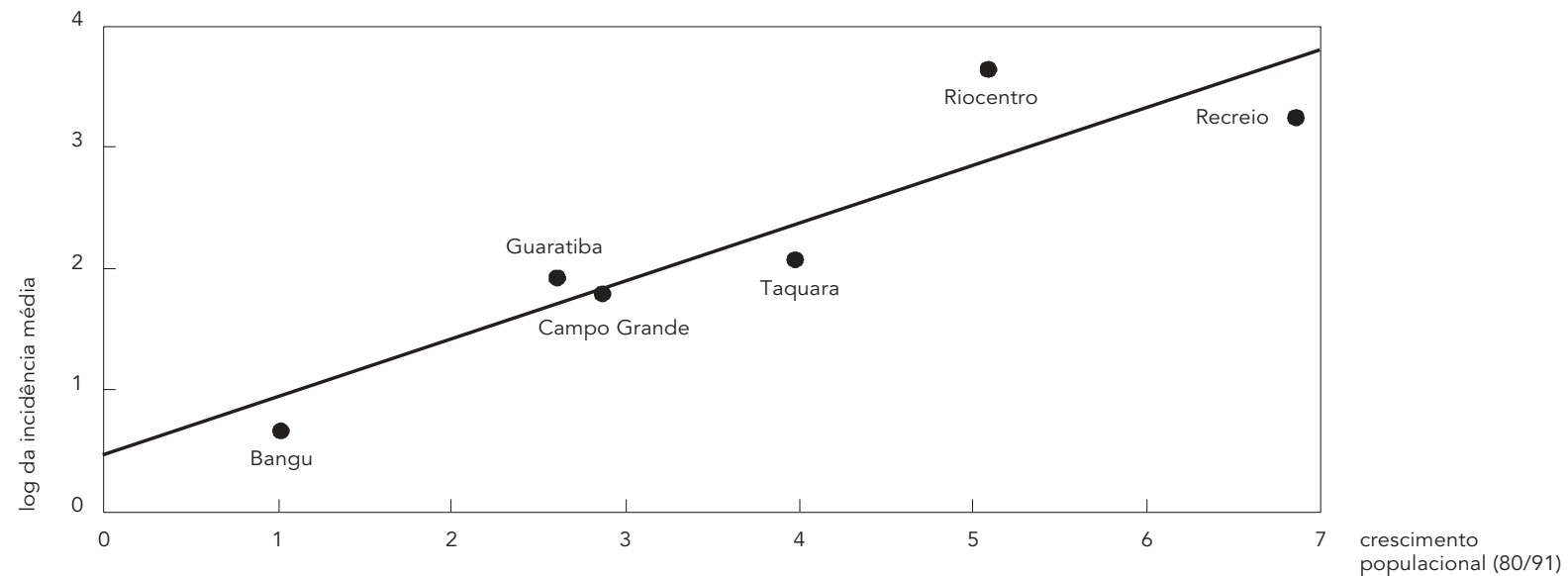

Fonte: Anuário Estatístico da Cidade do Rio de Janeiro, 1993/1994; Banco de Dados do Departamento de Endemias Samuel Pessoa da Escola Nacional de Saúde Pública da Fundação Oswaldo Cruz.

vamente ocorre desde 1870 na zona urbana da cidade...".

Esse novo modo de ocupação foi conduzido por diferentes lógicas de distintos agentes sociais modeladores do espaço urbano, levando a um constante processo de reorganização espacial.

Mediados por um conjunto de políticas públicas direcionadas a apoiar os investimentos, três vetores de penetração urbana redefiniram o modo de ocupação na região do Maciço da Pedra Branca, possibilitando a produção e reprodução do valor da terra nessas áreas e a construção de uma franja rural-urbana com características muito particulares.

$\mathrm{Na}$ direção norte, na primeira metade do século $X X$, um vetor industrial transformou de maneira irreversível aquela antiga zona rural, permitindo, a partir da local ização diferenciada das indústrias e vias de acesso, a formação e 
Figura 5

Densidade populacional e incidência média de leishmaniose tegumentar em áreas endêmicas.

Município do Rio de Janeiro, 1974 a 1988.

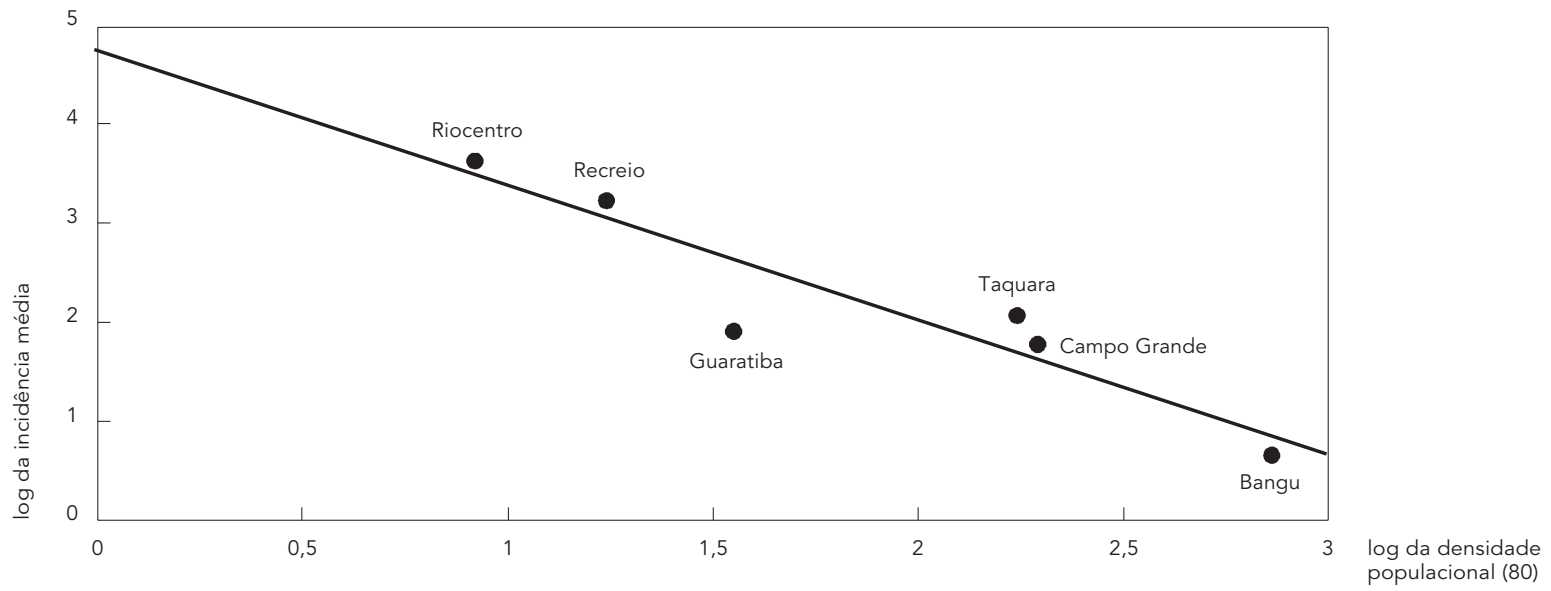

Fonte: Anuário Estatístico da Cidade do Rio de Janeiro, 1993/1994; Banco de Dados do Departamento de Endemias

Samuel Pessoa da Escola Nacional de Saúde Pública da Fundação Oswaldo Cruz.

consolidação da extensa área suburbana ao Iongo e em torno da estrada de ferro Central do Brasil e linhas auxiliares.

A decadência da citricultura de exportação devido à guerra, a combinação dos meios de transportes ferroviários e rodoviários, a abertura da Avenida Brasil em 1946 e da Rodovia Presidente Dutra em 1951, contribuíram ainda mais para intensificar a ocupação desses antigos subúrbios, transformando as propriedades rurais em loteamentos suburbanos como ocorreu em Campo Grande que, entre 1940-1950, apresentou um dos mais altos crescimentos populacionais da cidade (70\%) (Soares, 1965).

Nas áreas mais próximas do Maciço, distantes portanto dos eixos de integração rodo-ferroviária, as condições de ocupação se mantiveram quase inalteradas por décadas, tanto nas vertentes de Campo Grande como também de Jacarepaguá e Guaratiba, conforme descrito por Bernardes (1962:272): “Tem-se assi m esse fato insólito, eà primeira vista incompreensível, de que em alguns lugares do estado da Guanabara as abas dos morros, junto às estradas que cortam a baixada em vários sentidos, estão desocupadas, enquanto que, mais acima, nas vertentes de mais di fícil acesso, é que se vêem trechos cultivados, legíti mos quadros de ocupação rural. Assim ocorre, por exemplo, na serra do Quitungo, em algumas partes do Mendanha e, também no baixo vale do Pau da Fomeem Jacarepaguá".
Posteriormente, na década de sessenta, as grandes vias de expansão na direção sul da cidade, facilitam o acesso à área que corresponde atualmente a Jacarepaguá, Barra da Tijuca e suas imediações, através da abertura de túneis, viadutos e novas estradas viabilizando grandes empreendimentos imobiliários e transformando definitivamente a paisagem dessa região carioca.

O plano de industrialização da baixada de Jacarepaguá, que na década de setenta, trouxe profissionais relativamente especializados para as fábricas recentemente instaladas e também atraiu trabalhadores para o setor informal da economia, possibilitou o deslocamento de grande contingente populacional para essa região através desse circuito inferior integrado da economia, conceito utilizado por Santos (1978b).

Ao mesmo tempo, as leis de preservação ambiental que redefiniram o uso do solo nas áreas de encosta, impediram a construção de casas de al venaria e de atividades agrícolas permanentes nas altitudes acima de $100 \mathrm{~m}$, limitando a pressão de ocupação dessas áreas e preservando sua forma de ocupação tradicional.

Junto ao pólo dinâmico formaram-se, na Zona Oeste, áreas diferenciadas, mas integradas pelos mesmos processos sócio-espaciais, caracterizadas pela persistência de áreas com cobertura florestal e agricultura residual que foram mantidas, o que Santos (1978a:138) de- 
nominou rugosidades no espaço urbano: “As rugosi dades são o espaço construído, o tempo histórico que se transformou em pai sagem, incorporado ao espaço".

A função desempenhada por essas áreas com características rurais ou de reserva florestal no Rio de Janeiro foi a de reserva espacial, decorrente da expectativa de valorização da terra e de sua utilização especulativa, passando a constituir a franja urbano-rural da cidade.

Com a melhoria do acesso à Zona Oeste na década de sessenta, essa franja urbana, localizada nas vertentes, abaixo da cota de $100 \mathrm{~m}$, passou a ser ocupada por habitações do subproletariado do circuito inferior (Santos, 1978b) e por loteamentos residenciais de camadas médias do proletariado, que para ali se deslocaram em busca de oportunidades no mercado de trabalho, além de sítios de veraneio da classe média alta.

A implementação de novos modos de uso do solo nessas áreas fez com que a pressão exercida pelo maior incremento populacional gerasse tensão nessa franja rural-urbana - ecótonos de transição - estabelecendo condições propícias ao crescimento das populações de Lu. intermedia em altas densidades no entorno dos domicílios, tornando essas habitações vulneráveis à transmissão da LT devido ao alto contato parasita-hospedeiro.

Essas áreas de transição ou ecótonos, identificadas pela forma de ocupação do espaço urbano, correspondem ao que Gomes descreveu com relação aos espaços onde há acentuado efeito marginal, ou seja, onde a fauna é mais rica e abundante do que nas biocenoses adjacentes e a Lu. intermedia atinge suas densidades mais elevadas (Gomes, 1979).

O grande aumento demográfico decorrente do processo de urbanização na Zona Oeste permitiu a entrada de grupos populacionais suscetíveis à LT nesses ambientes já com grande densidade de vetores, favorecendo e amplificando a transmissão da endemia nessas áreas, em focos circunscritos, situados nos lugares onde $o$ acesso foi facilitado.

A localização desses focos, orientada pela topografia acidentada da região e pela concentração dessa população próximo aos pontos de acesso aos meios de transporte coletivo, contribuiu também para a distribuição desigual da $L T$, evidenciada pela presença de unidades espaciais com riscos diferenciados, como também foi relatado por Souza et al. (1992).
As el evadas densidades de Lu. intermedia vão encontrar as populações dessas regiões sem experiência anterior com o parasita e com alta mobilidade entre as diferentes áreas, possibilitando maior disseminação desses parasitas, seja por animais utilizados na circulação de mercadorias, como cavalos e muares, ou pela amplificação do processo de enzootia nos reservatórios domiciliados (Aguilar et al., 1989; Marzochi, 1992; Marzochi \& Marzochi, 1994).

Concomitantemente à valorização das áreas planas e de baixa declividade observou-se também que, apesar das leis proibitivas, aqueles antigos agricultores se viram obrigados a morar em terrenos de menor valor situados próximo ou mesmo dentro de reservas florestais e, consequentemente, com piores condições de acesso, infra-estrutura e saneamento, evidenciando um fenômeno semelhante àquele relatado por Sabroza et al. (1992:67): “Esses grupos marginalizados, obrigados a se desl ocar para fora das áreas mais val orizadas, procuraram os locais menos acessíveis, ecótopos onde diferentes ciclos zoonóti cos estavam estabel ecidos e que vão sendo, então, modificados pela sua presença".

Todas essas transformações decorrentes da incorporação da Zona Oeste ao espaço urbano da cidade, produziram a nova região de endemicidade da LT no Rio de Janeiro, cuja configuração espacial se caracterizou pela presença de um conjunto de focos antropúrgicos restritos, articulados, delimitados pelo processo de urbanização e trabalho, tendo sua dinâmica determinada pela valorização do solo urbano.

Concluindo, observamos que as condições específicas da transmissão da LT na cidade do Rio de Janeiro, evidenciaram uma dinâmica espaço temporal muito particular, constituída por dois movimentos: a criação de uma estrutura em mosaico caracterizada pela pressão exercida por uma população com alta mobilidade territorial e pela expansão de um subproletariado urbano, com acesso facilitado pelos novos meios de transporte e, um segundo movimento, de preservação ambiental, que resultou na conservação da maior floresta tropical do mundo dentro de uma área urbana, com sua grande biodiversidade, que inclui a presença de diversos parasitas e seus vetores de doenças. Mediados pelo valor da terra, esses dois movimentos possibilitaram a produção e reprodução da LT e a emergência de uma nova região de endemicidade na Zona Oeste da cidade do Rio de Janeiro. 


\section{Referências}

ABREU, M. A., 1992. A cidade, a montanha e a floresta. In: Natureza eSociedade no Rio de Janeiro (M. A. Abreu, org.), pp. 54-103, Rio de Janeiro: Divisão de Editoração, Departamento de Documentação e Informação Cultural, Secretaria Municipal de Cultura, Turismo e Esportes, Prefeitura da Cidade do Rio de Janeiro.

AGUIAR, G. M.; MEDEIROS, W. M.; DE MARCO, T. S.; SANTOS, S. C. \& GAM BARDELLA, S., 1996. Ecologia dos flebotomíneos da Serra do Mar, Itaguaí, Estado do Rio de Janeiro, Brasil. I - A fauna flebotomínica e prevalência pelo local e tipo de captura (Diptera, Psychodidae, Phlebotominae). Cadernos de Saúde Pública, 12:195-206.

AGUILAR, C. M.; RANGEL, E. F.; GARCIA, L.; FERNANDEZ, E.; MOMEN, H.; GRIMALDI Jr., G. \& VARGAS, Z., 1989. Zoonotic cutaneous leishmaniasis due to Leishmania (Viannia) braziliensis associated with domestic animals in Venezuela and Brazil. Memórias do Instituto Oswal do Cruz, 84:19-28.

ALBUQUERQUE, M. F. P. M., 1993. Urbanização, favelas e endemias: A produção da filariose no Recife, Brasil. Cadernos de Saúde Pública, 9:487-497.

ANDRADE, L. S., 1998. Leishmaniose Tegumentar Americana em Área de Ocupação Recentena Periferia da Cidade de Manaus, Estado do Amazonas, Brasil. Dissertação de Mestrado, Rio de Janeiro: Instituto Oswaldo Cruz, Fundação Oswaldo Cruz.

ARAGÃO, H. B., 1922. Transmissão da leishmaniose no Brasil pelo Phlebotomus intermedius. BrasilMédico, 36:129-130.

AZEVEDO, R. V., 1984. A Percepção Popular da Leishmaniose Tegumentar Americana: Estudo Sorológico Realizado junto a Famílias de Baixa Renda da Localidade do Pau da Fome (Jacarepaguá, Rio de Janeiro). Dissertação de Mestrado, Rio de Janeiro: Escola Nacional de Saúde Pública, Fundação Oswaldo Cruz.

BARBOSA-SANTOS, E. G. O.; MARZOCHI, M. C. A.; CONCEIÇÃO, N. F.; BRITO, C. M. M. \& PACHECO, R. S., 1998. Epidemiological survey on canine population with the use of immunoleish skin test in endemic areas of human american cutaneous leishmaniasis in the state of Rio de Janeiro, Brazil. Revista do Instituto de Medicina Tropical de São Paulo, 40:41-47.

BERNARDES, N., 1962. Notas sobre a ocupação humana da montanha no estado da Guanabara. In: Aspectos da Geografia Carioca (Associação dos Geógrafos Brasileiros, org.), pp. 187-210, Rio de Janeiro: Associação dos Geógrafos Brasileiros.

CEROUEIRA, A. C. \& VASCONCELLOS, A., 1922. A Leishmaniose nesta capital. Boletim Sanitário. Publicações Científicas do Departamento Nacional de Saúde Pública, 1:35-47.

CORRÊA, R. L., 1987. Região e Organização Espacial São Paulo: Editora Ática.

CORRÊA, R. L., 1992. O meio ambiente e a metrópole. In: Natureza e Sociedade no Rio de Janeiro (M. A. Abreu, org.), pp. 27-36, Coleção Biblioteca Carioca. Rio de Janeiro: Divisão de Editoração, Departamento Geral de Documentação e Informação Cultural. Secretaria Municipal de Cultura, Turis- mo e Esportes, Prefeitura da Cidade do Rio de Janeiro.

d'UTRA-e-SILVA, O., 1915. Sobre a leishmaniose tegumentar e seu tratamento. Memórias do Instituto Oswaldo Cruz, 7:213-248.

GOMES, A. C., 1979. Observações Ecológicas sobre Psychodygus intermedius no Vale do Ribeira, Estado de São Paulo, Brasil. Tese de Doutorado, São Paulo. Universidade de São Paulo.

GOMES, A. C. \& NEVES, V. L. F. C., 1998. Estratégia e perspectivas de controle da leishmaniose tegumentar no Estado de São Paulo. Revista da Sociedade Brasileira de Medicina Tropical, 31:553-558.

GRIM ALDI J r., G.; TESH, R. B. \& MCM AHON-PRATT, D., 1989. A review of the geographic distribution and epidemiology of leishmaniasis in the New World. American Journal of Tropical Medicine and Hygiene, 41:687-725.

IPLANRIO (Empresa Municipal de Informática e Planejamento), 1995. Anuário Estatístico da Cidade do Rio de Janeiro 93/94. Rio de Janeiro: Prefeitura da Cidade do Rio de Janeiro.

KAWA, H., 1998. Organização do Espaço e Produção da Leishmaniose Tegumentar no Município do Rio deJaneiro. Dissertação de Mestrado, Rio de Janeiro: Instituto de Medicina Social, Universidade do Estado do Rio de Janeiro.

LIMA, L. C., 1986. Ruralização da Lutzomyia interme dia: Um provável caso de pré-adaptação. Revista deSaúde Pública, 20:102-104.

MARZOCHI., M. C. A., 1985. A survey for American cutaneous and visceral leishmaniasis among 1,342 dogs from areas in Rio (Brazil) where the human diseases occur. Memórias do Instituto Oswaldo Cruz, 80:17-22.

M ARZOCHI, M. C. A., 1992. Leishmanioses no Brasil: As leishmanioses tegumentares. Jornal Brasileiro de Medicina, 63:82-104.

MARZOCHI, M. C. \& MARZOCHI, K. B. F., 1994. Tegumentary and visceral leishmaniases in Brazil Emerging anthropozoonosis and possibilities for their control. Cadernos deSaúde Pública, 10(Sup. 2):359-375

MS (Ministério da Saúde), 2000. Manual de Controle da Leishmaniose Tegumentar Americana. Brasília: MS

OLIVEIRA NETO, M. P.; PIRMEZ, C.; RANGEL, E.; SCHUBACH, A. \& GRIMALDI Jr., G., 1988. An oubreak of american cutaneous leishmaniasis (Leishmania braziliensis braziliensis) in a periurban area of Rio de Janeiro city, Brazil: Clinical and epidemiological studies. Memórias do Instituto Oswaldo Cruz, 83:427-435.

PAVLOVSKY, E. N., 1964. Natural Nidality of Transmissible Diseases. Moscow: Peace Publishers.

PESSOA, S. B. \& BARRETO, M. P., 1948. Leishmaniose Tegumentar Americana. Rio de Janeiro: Serviço de Documentação, Ministério da Educação e Saúde.

PIRMEZ, C., 1986. Leishmaniose Tegumentar Americana Canina. Estudo Clínico, Histopatológico, e Sorológico da Infecção Natural e Experimental por L. b. braziliensis. Dissertação de Mestrado, Rio de Janeiro: Universidade Federal do Rio de Janeiro. 
RABELLO, E., 1913. Caso de leishmaniose. Boletim da Sociedade Brasileira de Dermatologia, 2:72.

RANGEL, E. F.; AZEVEDO, A. C. R.; ANDRADE, C. A.; SOUZA, N. A. \& WERMELINGER, E. D., 1990. Studies on sandfly fauna (Diptera: Psychodidae) in a foci of cutaneous leishmaniasis in Mesquita, Rio de Janeiro, Brazil. Memórias do Instituto Oswaldo Cruz, 85:39-45.

RIBEIRO, L. C. Q., 1997. Dos Cortiços aos Condomínios Fechados - As Formas de Produ ção da Moradia na Cidade do Rio de Janeiro. Rio de Janeiro: Civilização Brasileira.

ROSICKY, B., 1967. Natural foci of diseases. In: Infectous Diseases. Their Evolution and Eradication (A. Cockburn, ed.), pp. 108-126, Springfield: Charles C. Thomas Publisher.

SABROZA, P. C., 1981. O Domicílio como Fator de Risco na Leishmaniose Tegumentar Americana: Estudo Epidemiológico em Jacarepaguá, Município do Rio de Janeiro. Dissertação de Mestrado, Rio de Janeiro: Escola Nacional de Saúde Pública, Fundação Oswaldo Cruz.

SABROZA, P. C., KAWA, H., \& CAM POS, W. S. Q., 1995. Doenças transmissíveis: Ainda um desafio. In: Os Muitos Brasis - Saúde e População na Década de 80 (M. C. S. Minayo, org), pp. 177-244, São Paulo: Editora Hucitec/Rio de Janeiro: ABRASCO.

SABROZA, P. C.; TOLEDO L. M. \& OSANAI, C. H., 1992. A organização do espaço e os processos endêmico-epidêmicos. In: Saúde, Ambiente e Desenvolvimento (M. C. Leal, P. C. Sabroza, R. H. Rodriguez \& P. M. Buss, org.), v. II, pp. 57-77, Rio de Janeiro: ABRASCO/São Paulo: Editora Hucitec.

SANTOS, M., 1978a. Por uma Geografia Nova; Da Crítica da Geografia a uma Geografia Crítica. São Paulo: Editora Hucitec.

SANTOS, M., 1978b. O Espaço Dividido. Os Dois Circuitos da Economia Urbana dos Países Subdesenvolvidos. Rio de Janeiro: Livraria Francisco Alves Editora.
SANTOS, M., 1988. Metamorfoses do Espaço Habitado. São Paulo: Editora Hucitec.

SILVA, L. J., 2000. A ocupação do espaço e a ocorrência de endemias. In: Doenças Endêmicas: Abordagens Sociais, Culturais e Comportamentais (R. B. Barata \& R. Briceño-León, org.), pp. 139-150, Rio de Janeiro: Editora Fiocruz.

SINNECKER, H., 1976. General Epidemiology. London: John Wiley \& Sons.

SOARES, M.T. S., 1965. Fisionomia e estrutura do Rio de Janeiro. Revista Brasileira de Geografia, 27:329387.

SOARES, V. B.; CARVALHO, R. W.; FERNANDES, O.; PIRMEZ, C. \& SABROZA, P. C., 2000. Estudo de infecção por Leishmania em roedores durante surto de LTA. In: VI Congresso Brasileiro de Saúde Coletiva, Resumos, p. 431. Salvador: ABRASCO.

SOARES, V. B.; SABROZA, P. C. \& KAWA, H., 1995. Padrões de transmissão da leishmaniose tegumentar no estado do Rio de Janeiro. In: III Congresso Brasileiro, II Congresso Ibero-Americano, I Congresso Latino Americano de Epidemiologia, Anais, p. 282. Salvador: ABRASCO.

SOUZA, W. J. S.; SABROZA, P. C.; SANTOS, C. S.; SOUSA, E.; HENRIQUE, M. F. \& COUTINHO, S. G., 1992. Montenegro skin tests for American cutaneous leishmaniasis carried out on school children in Rio de Janeiro, Brazil: An indicator of transmission risk. Acta Tropica, 51:111-119.

TOLEDO, L. M., 1987. Leishmaniose Tegumentar e Leishmaniose Visceral em Área Peri-Urbana no Município do Rio de Janeiro. Dissertação de Mestrado, Rio de Janeiro: Instituto Oswaldo Cruz, Fundação Oswaldo Cruz.

VALIM, C., 1993. Transmissão da Leishmania (Viannia) braziliensis no Ceará. Dissertação de Mestrado, Rio de Janeiro: Escola Nacional de Saúde Pública, Fundação Oswaldo Cruz.

Recebido em 23 de julho de 2001

Versão final reapresentada em 3 de dezembro de 2001 Aprovado em 7 de março de 2002 